\title{
Are Glucose Readings Sufficient to Adjust Insulin Dosage?
}

\author{
Eran Bashan, Ph.D., William H. Herman, M.D., M.P.H., ${ }^{2}$ and Israel Hodish, M.D., Ph.D.,2
}

\begin{abstract}
Aims/hypothesis: Insulin therapy is effective predominantly when dosage is frequently adjusted. However, a controversy surrounds the pertinent clinical parameters required to make effective and safe frequent dosage adjustments. We hypothesize that glucose readings are sufficient to adjust insulin dosage provided that dosage is adjusted every 1-4 weeks.

Methods: To test the hypothesis, we generated several algorithms implemented in software to process glucose readings and recommend insulin dosage adjustments. A post hoc analysis was made on $630 \log$ sheets $(2,520$ insulin dosage adjustments) from 26 older adults with suboptimally controlled type 2 diabetes. The subjects were followed for a year and treated with intensive insulin therapy that was titrated every $1-4$ weeks by a trained study team. More than $88 \%$ of subjects attained the treatment goal (hemoglobin A1c $<7 \%$ ) without excessive hypoglycemia. Glucose readings from each log sheet were used as an input to the software, and its recommendations for insulin dosage adjustments were compared to the original ones made by the study team. While the study team could have been exposed to multiple clinical parameters, the software relied solely on glucose readings.

Results: The software recommendations for dosage adjustments were clinically equivalent to the original study team's recommendations in more than $95 \%$ of the cases, unrelated to patients' insulin sensitivity. The remaining $4.4 \%(n=111)$ were thoroughly examined, yet we did not find any recommendations suggested by the software to be unsafe or unreasonable.

Conclusions/Interpretation: Glucose readings are sufficient to effectively adjust insulin dosage provided that adjustments are made every 1-4 weeks. Therefore, dedicated software can help adjusting insulin dosage between clinic visits.
\end{abstract}

\section{Introduction}

I

NSULIN IS THE ONLY ANTIDIABETES agent lacking a therapeutic window. In other words, most insulin-treated patients can achieve satisfactory glycemic control provided that appropriate formulations and adequate dosage are prescribed. Yet, almost two-thirds of insulin-treated diabetes patients in the United States fail to reach the therapy goal (hemoglobin A1c [A1C] $<7 \%$ ), ${ }^{1,2}$ although compliance to antidiabetes medications is generally considered adequate in about three-fourths of the cases. ${ }^{3,4}$ Most insulin users have type 2 diabetes; some use partial insulin therapy (premixed, biphasic, or long-acting insulin), and some use intensive insulin therapy (basal/bolus). Only clinical trials that reinforce a policy of insulin dosage adjustments every 1-4 weeks (cumulatively $>15,000$ patient-years) for either partial ${ }^{5-10}$ or intensive insulin therapy ${ }^{11-13}$ achieved treatment goals (A1C $<7 \%$ ) among $40 \%$ and $80 \%$ of the subjects, respectively. Al- though this finding is hard to separate from the Hawthorne effect, when trials have ended and frequent insulin dosage adjustment strategy was replaced with infrequent conservative care predominantly during clinic appointments, patients' A1C was unfavorable merely a year after study termination. $^{13,14}$

The nature of this paradox likely ensues from patients' metabolic behavior. Because of variability within each patient (Fig. 1), insulin requirements significantly fluctuate over time and thus cannot be met by infrequent dosage adjustments during clinic visits (typically once every 3-6 months).

Most clinicians agree that insulin dosage should be titrated/ adjusted far more frequently than during routine clinic visits. However, the clinical parameters as well as the frequency required to make effective frequent dosage adjustments are still controversial. Insulin dosage should not be confused with insulin doses that for some regimens may depend on preprandial glucose reading (sliding scales or correction factors)

\footnotetext{
${ }^{1}$ Hygieia, Inc., Ann Arbor, Michigan.

${ }^{2}$ Division of Metabolism, Endocrinology and Diabetes, Department of Internal Medicine, University of Michigan Medical Center, Ann Arbor, Michigan.
} 


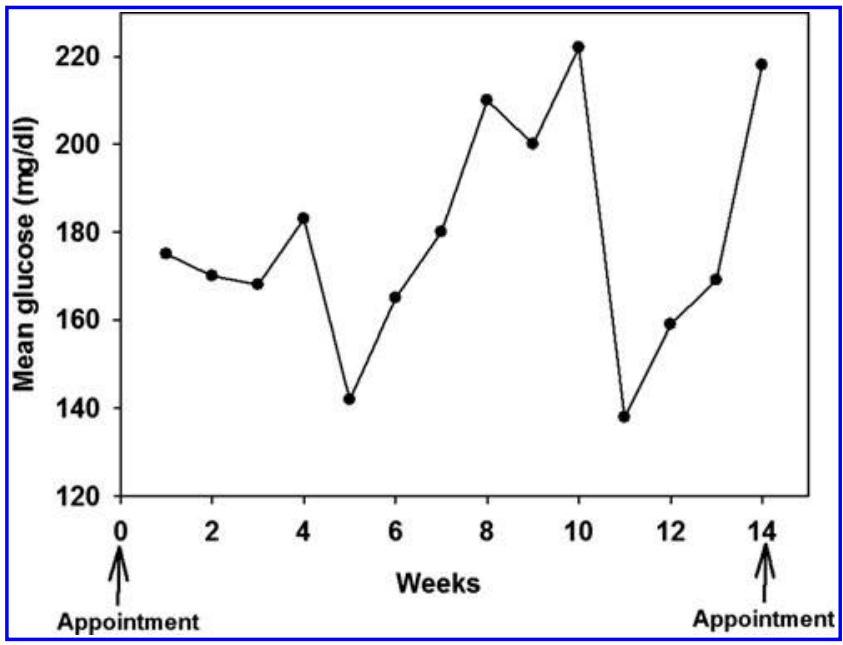

FIG. 1. Weekly mean glucose levels between clinic visits of a type 2 diabetes patient with uncontrolled diabetes. This patient did not take part in the discussed study. Significant fluctuations in mean glucose levels between visits make dosage adjustment only during clinical visits inadequate.

and carbohydrate intake. A common convention is that diet and exercise habits need to be explored before insulin dosage could be adjusted. Some believe that dosage should be adjusted every 3 days or even daily. Others believe that carbohydrate counting is crucial for the success of the regimen. However, it has been established that for patients with type 2 diabetes treated with intensive insulin therapy, carbohydrate counting is not superior to a sliding scale provided that dosage is frequently adjusted. ${ }^{12}$

Many of the aforementioned studies also published the instructions given to the study team for frequent insulin dosage titrations. These instructions advised on frequent insulin dosage adjustments solely based on glucose readings. ${ }^{5,7-10,12}$ Yet, because these instructions encourage considerable amount of flexibility and clinical judgment, it is unclear how faithfully they were followed. ${ }^{15,16}$

We hypothesize that glucose readings are sufficient to effectively adjust insulin dosage provided that dosage is adjusted every 1-4 weeks. To test the hypothesis, we generated several algorithms and implemented them in software to recommend insulin dosage adjustments based only on glucose readings. Glucose readings from log sheets of subjects treated with intensive insulin therapy that was titrated every 1-4 weeks by a trained study team were used as an input to the software. While the study team could have been exposed to multiple clinical parameters, the software relied solely on glucose readings. The software and the study team's recommendations for insulin dosage adjustments were systematically compared using a similarity metric.

\section{Subjects and Methods}

\section{Original study design}

The original study was designed as a two-center, prospective, randomized, controlled, clinical trial. Inclusion and exclusion criteria are available elsewhere.$^{11}$ In brief, subjects were $\geq 60$ years of age, had a clinical diagnosis of type 2 diabetes for at least 1 year, were taking at least one injection of insulin per day (with or without oral antidiabetes medications), and had an A1C $\geq 7.0 \%$. One hundred seven subjects were randomized, and 98 completed follow-up. Patients were randomized to either multiple daily insulin injections or continue subcutaneous insulin injections. Of the 54 patients randomized to multiple daily insulin injections, data from 28 subjects who were followed at the Michigan site were available for re-analysis. Data from two of the 28 patients were incomplete and insufficient for re-analysis. The remaining 26 subjects were cumulatively followed for 22 patient-years.

The multiple daily insulin injection regimen consisted of once-daily insulin glargine (Lantus ${ }^{\circledR}$, Aventis, Bridgewater, NJ) and preprandial insulin lispro (Humalog ${ }^{\circledR}$, Eli Lilly, Indianapolis, IN). All subjects were instructed to monitor their blood glucose levels before meals and at bedtime. At least once a week, subjects were instructed to monitor nocturnal blood glucose levels. Initial basal insulin dosage was calculated as $50 \%$ of the total daily insulin dose and administered as glargine before bedtime. The remaining 50\% was administered as preprandial lispro boluses and formulated as a sliding scale. Accordingly, dosage was given as a combination of four elements: (1) basal insulin dose, (2) sliding scale for breakfast, (3) sliding scale for lunch, and (4) sliding scale for dinner. Dosage adjustments were made once every 1-4 weeks by the study team, which included three endocrinologists and two nurses with extensive experience in intensive insulin therapy. Subjects submitted log sheets (for an example see Supplementary Appendix; Supplementary Data are available online at www.liebertonline.com/dia) including dosage given by the study team, time-tagged glucose reading, and insulin doses. The study team provided a new recommended dosage on each log sheet, which was later communicated to the subject via the study coordinator.

Efficacy was assessed by A1C measured at the baseline and at the 1-, 2-, 4,- 6-, 8-, 10-, and 12-month visits. Safety was evaluated by the rate of severe hypoglycemia, defined as capillary glucose $\leq 2.8 \mathrm{mmol} / \mathrm{L}(\leq 50 \mathrm{mg} / \mathrm{dL})$ associated with neuroglycopenic symptoms that require assistance by another person. Twenty-three of the 26 subjects successfully and safely reached $\mathrm{A} 1 \mathrm{C}<7 \%$ (more than $88 \%$ ), and mean $\mathrm{A} 1 \mathrm{C}$ improved from $7.8 \%$ to $6.3 \%$.

\section{Determination of adherence}

Subjects' adherence served as a marker for quality control to corroborate the weight of the study team's instructions. For each patient, lispro meal doses over a period of two randomly chosen weeks were individually compared to the sliding scale of the fast-acting insulin boluses. The deviation of each administered dose from the expected dose due to the sliding scale was expressed as a percentage.

\section{Software design}

To prove or refute our hypothesis it was mandatory to refrain from human interference while regenerating dosage recommendations and comparing them to the study team's recommendations. Therefore, we created software with algorithms that processed time-tagged glucose readings and adjusted insulin dosage while ignoring any additional clinical parameters. The software was developed by E.B. and I.H. prior to acquiring the clinical data. W.H.H. and his study team, from whom the data were acquired, ${ }^{11}$ were not in- 
volved in the development of the software. The algorithms embedded in the software were based on state-of-the-art guidelines for insulin management ${ }^{17}$ and on the following four principles:

1. Time-tagged glucose readings were the only input used to adjust the current dosage and create recommendations for the next insulin dosage.

2. Insulin dosage was increased for glucose level above target and decreased for glucose level below target.

3. The intensity of adjustments decreases as glucose readings get closer to target to prevent unstable oscillations of dosage.

4. The ability to detect "outliers." The software utilizes higher-order statistics to detect outliers and treats them separately from the remainder of the data.

\section{Similarity between the software and study team's recommendations}

The database consisted of $630 \log$ sheets (2,520 insulin dosage adjustments) containing time-tagged glucose readings, previous dosage prescribed by the study team, and a new set of adjusted dosage (see Supplementary Appendix). Each log sheet's glucose readings were processed by the software. The software generated new recommended dosage for long-acting insulin and three mealtime fast-acting insulin sliding scales. Because of inherent dependencies, only the $4.4-6.7 \mathrm{mmol} / \mathrm{L}(80-120 \mathrm{mg} / \mathrm{dL})$ glucose range of each sliding scale, i.e., each meal bolus dose, was used for comparison. These four components generated by the software per log sheet were compared to the original study team's recommendations using a similarity metric.

\section{Similarity metric}

Differences between the study team recommendations and the software recommendations were classified into six categories:

1. Identical: the software and study team made the same dosage recommendation.

2. Within $10 \%$ : the dosage recommended by the software was within $10 \%$ of the dosage recommended by the study team. In addition, the two dosage modifications were in the same direction (i.e., the software did not recommend increasing the dosage, whereas the study team recommended decreasing the dosage, or vice versa).

3. Within $10-20 \%$ : the dosage recommended by the software was within $10-20 \%$ of the dosage recommended by the study team. In addition, the two dosage modifications were in the same direction.

4. Different; 10-20\%: The software recommended increasing the dosage by $10-20 \%$, whereas the study team recommended decreasing the dosage.

5. Different; more than 20\%: The software recommended increasing the dosage by more than $20 \%$, while the study team recommended decreasing the dosage.

6. Other: all other cases (not complying with categories $1-5)$.

Of the above, categories 4 and 5 were defined as "Different" and represented potentially hazardous disagreement between the software and the study team.

We considered the software recommendations to be "clinically equivalent" to the study team's recommendations if they were classified in categories 1,2 , or 3 . We assumed that a $20 \%$ difference is a reasonable value that can be seen in routine

Table 1. Subjects' Characteristics Compared to the Original Randomized Study Population

\begin{tabular}{|c|c|c|}
\hline Characteristic & $\begin{array}{l}\text { Multiple daily insulin } \\
\text { subjects for re-analysis }\end{array}$ & $\begin{array}{c}\text { Original randomized } \\
\text { population }\end{array}$ \\
\hline Subjects $(n)$ & 26 & 107 \\
\hline Age (years) & $67.4 \pm 4.9$ & $66.2 \pm 5.4$ \\
\hline Sex (male) & $13(50)$ & $62(58)$ \\
\hline \multicolumn{3}{|l|}{ Race } \\
\hline Caucasian & $26(100)$ & $92(86)$ \\
\hline Black & 0 & $6(5.6)$ \\
\hline Hispanic & 0 & $6(5.6)$ \\
\hline Other & 0 & $3(2.8)$ \\
\hline Duration of diabetes (years) & $18.3 \pm 9.9$ & $15.9 \pm 9.0$ \\
\hline \multicolumn{3}{|l|}{ History of diabetes complications } \\
\hline Retinopathy & $9(34.6)$ & $39(36)$ \\
\hline Nephropathy & $5(19)$ & $16(15)$ \\
\hline Neuropathy & $14(53.8)$ & $69(64)$ \\
\hline \multicolumn{3}{|l|}{ History of cardiovascular complications } \\
\hline Hypertension & $19(73)$ & $79(74)$ \\
\hline Dyslipidemia & $19(73)$ & $72(67)$ \\
\hline Cigarette smoking (current) & $1(4)$ & $5(5)$ \\
\hline Ischemic heart disease/heart failure & $10(38)$ & $38(35)$ \\
\hline BMI $\left(\mathrm{kg} / \mathrm{m}^{2}\right)$ & $29.9 \pm 6.1$ & $32.2 \pm 5.7$ \\
\hline A1C (\%) & $7.8 \pm 0.7$ & $8.2 \pm 1.0$ \\
\hline
\end{tabular}

Data are mean $\pm \mathrm{SD}$ values or $n(\%)$.

No statistically significant differences were noted.

$\mathrm{A} 1 \mathrm{C}$, hemoglobin A1c; BMI, body mass index. 

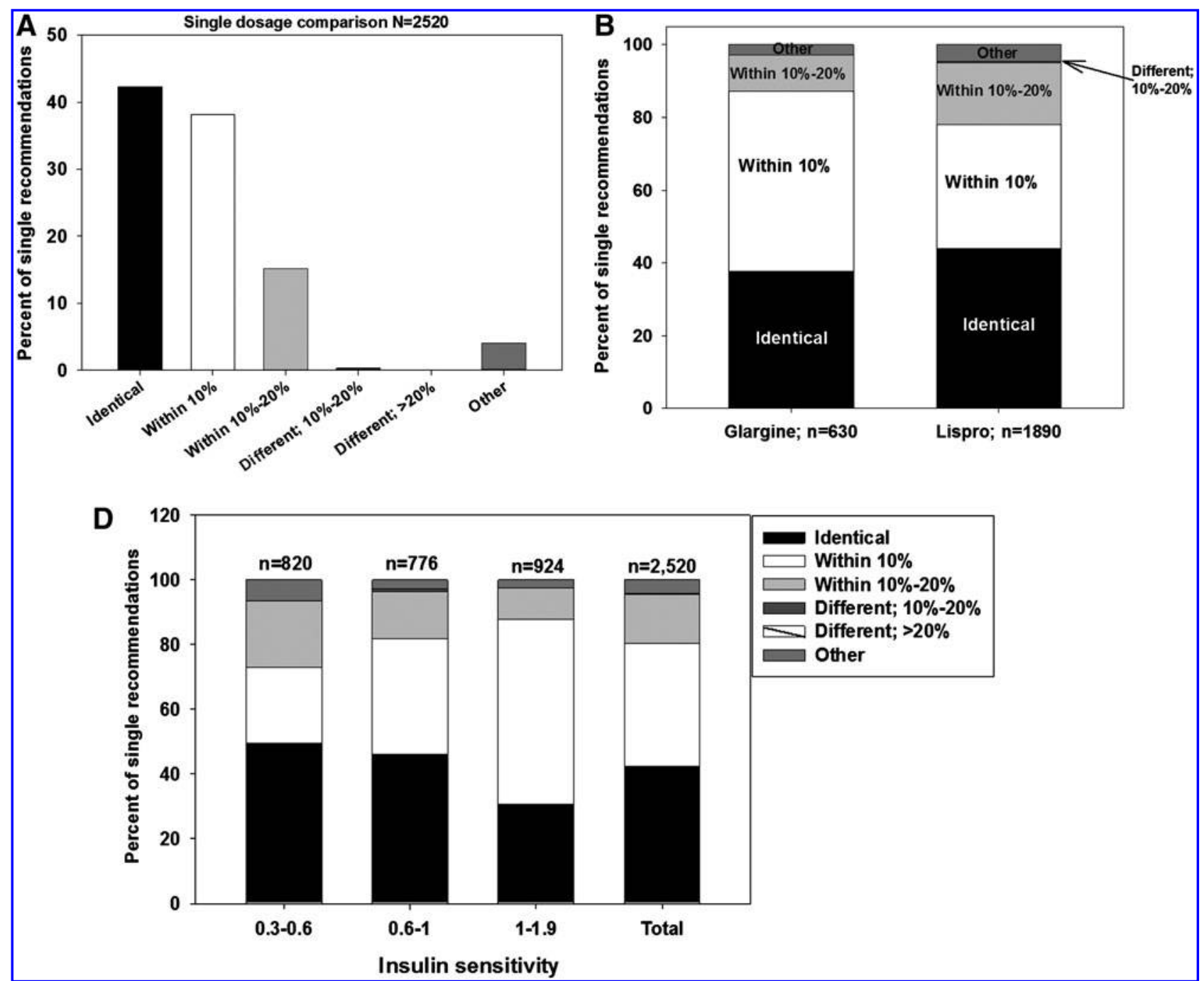

FIG. 2. Similarity between the software and study team's dosage modifications. (A) Overall similarity between the software and the study team's dosage recommendations. (B) Similarity between dosage recommendations analyzed independently for long-acting insulin (glargine) and fast-acting insulin (lispro). (C) Example of two subjects. The subjects' hemoglobin A1c (HgbA1c) levels dropped below 7\% in less than 10 weeks, yet dosage kept changing to keep HgbA1c within the desired range. Discrete points in the upper plot represent the software dosage recommendations compared to the study team's recommendations depicted in the curves. (D) Distance between recommendations made by the software and the study team for subjects with different insulin sensitivity (total daily insulin [in units]/weight [in $\mathrm{kg}$ ]). The population was analyzed for subjects who had high (0.3-0.6), medium (0.6-1), or low (1-1.9) insulin sensitivity. The clinical similarity between dosage modifications made by the software and the study team was not affected by insulin sensitivity.

clinical settings. Moreover, it has been demonstrated that identical insulin injections can result in different plasma insulin profiles (can exceed $20 \%$ ) because of the complex process of insulin absorption and dispersion. ${ }^{18}$ For illustration, we believe that it is not unreasonable in clinical settings to have one care-provider increasing lunchtime lispro from 20 units to 21 units and another from 20 units to 24 units, using data from the same patient. The difference between these two examples is almost $20 \%$.

\section{Statistical analysis}

Subjects' characteristics were compared to the original study population by two-tailed Student's $t$ test for parametric variables and $\chi^{2}$ test for nonparametric variables. Results are presented as mean \pm SD values. A $P$ value $<0.05$ was defined as statistically significant.
The process of insulin dosage recommendations, by either the study team or the software, is not a random process. Therefore, we neither performed nor presented statistical comparisons between the software and study team's recommendations. Instead, the similarity metric was used to measure the distance between the two sets.

\section{Results}

The re-analyzed subjects represent the original study population

Data from the 26 subjects treated with multiple daily insulin injections were available for post hoc analysis. Table 1 compares the basic characteristics of this cohort to the original study population that was eligible for 


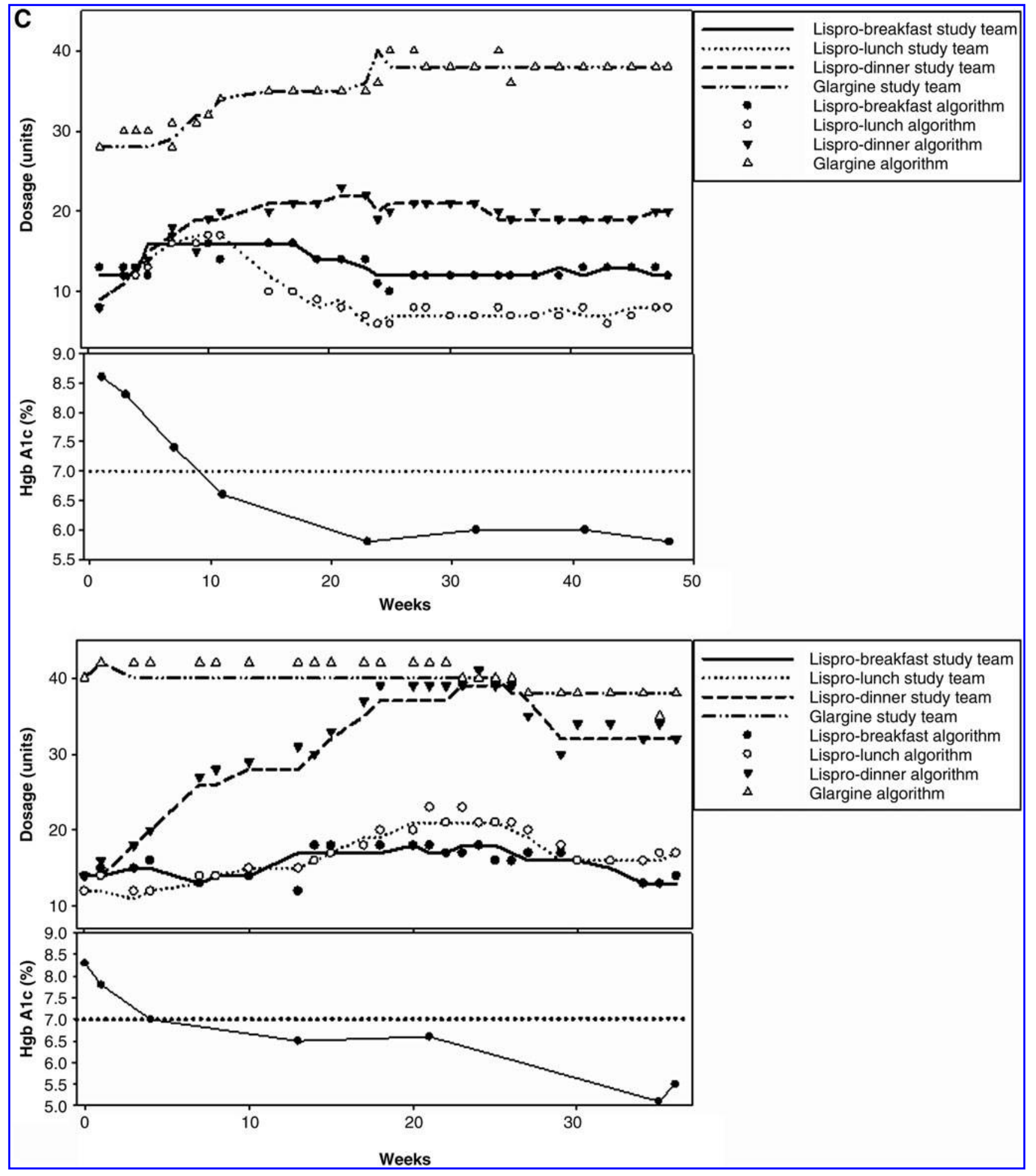

FIG. 2. (Continued).

randomization $(n=107)$. No significant differences were identified.

Mean \pm SD A1C of the re-analyzed subjects was $7.8 \pm 0.7 \%$ before enrollment (median, 7.7\%), and following 12 months of intensive insulin therapy it was $6.3 \pm 0.8 \%$ (median, $6.1 \%)(P<0.0001)$. The mean improvement $( \pm \mathrm{SD})$ of $\mathrm{A} 1 \mathrm{C}$ was $1.5 \pm 0.9 \%$ (median, $1.6 \%$ ) and was similar to that of the original study population. Mean total daily insulin dosage of the re-analyzed subjects was 66.2 units $(0.7$ units $/ \mathrm{kg})$ at the beginning of the study and 104.5 units $(1.0$ units $/ \mathrm{kg})$ at the study's end. Among the re-analyzed subjects $88.5 \%$ (23 of 26) achieved $\mathrm{A} 1 \mathrm{C}<7 \%$ at the expense of six episodes of severe hypoglycemia ( 0.27 events per person-year). Ninety-six percent of the re-analyzed patients had at least one episode of 
minor hypoglycemia. The frequency of these events was not statistically different from that observed in the original study population (in the original study $90 \%$ of patients incurred minor hypoglycemia, and the frequency of severe hypoglycemia was 0.16 events per person-year) and was comparable to that reported in subjects treated with intensive insulin therapy (most available data derive from patients with type 1 diabetes ${ }^{19-21}$ ). Body mass index of the 26 subjects did not significantly change during the study (initial value, $29.9 \pm 6.1 \mathrm{~kg} / \mathrm{m}^{2}$; final value, $30.9 \pm$ $\left.6.5 \mathrm{~kg} / \mathrm{m}^{2}\right)$.

\section{Subjects were adherent to the study team's instructions}

To determine dosing accuracy, 1,239 meal boluses were compared to the corresponding sliding scales. Each deviation from a prescribed dose was expressed as a percentage. For instance, if for a pre-lunch glucose level of $10 \mathrm{mmol} / \mathrm{L}$ $(180 \mathrm{mg} / \mathrm{dL})$ a subject was instructed to administer 24 units of lispro and instead injected 26 , this dose was registered as $108.3 \%$. In $77.2 \%$ of the instances the reported dose was identical to the prescribed one. In $13.4 \%$ of the instances the reported dose deviated from the prescribed dosage by more than $10 \%$ (data not shown). We assumed that in these cases, subjects deviated from the prescribed dose because of factors such as decreased food intake or increased physical activity.

\section{Although incorporating only glucose readings, the software recommendations lie in close proximity to those recommended by the study team}

A database of $n=2,520$ recommendations was available for comparison. As shown in Figure 2A, the software made "Identical" (similarity metric category 1 ) dosage recommendations (long-acting and fast-acting insulin) in $42.3 \%$ $(n=1,067)$ of the cases, $38 \%(n=959)$ were "Within $10 \%$ " (category 2), and 15.2\% $(n=383)$ were "Within 10-20\%" (category 3).

When analyzed separately, both long-acting (glargine) and fast-acting (lispro) gave identical or similar recommendations in $95-97 \%$ of the cases, respectively (Fig. 2B). Eight cases $(0.3 \%)$ were considered "Different; $10-20 \%$ " (category 4$)$. Of these eight, seven cases were recorded in a single subject treated with 51 units of insulin/day. These seven cases represented $7.95 \%$ of the 88 dosage recommendations for this particular subject. In all of these cases, the study team recommended decreasing the dosage by a single unit, whereas the software recommended increasing the dosage by a single unit. The eighth case was recorded in a patient treated with 55 units of insulin/day. In this particular event, the study team recommended decreasing the patient's lunch lispro dosage from 11 to 10 insulin units, whereas the software recommended increasing the same dosage from 11 to 13 insulin units. Pre-dinner glucose measurements for the discussed week were $8,10,20.3,4.4$, and $18.7 \mathrm{mmol} / \mathrm{L}(144,180,365,79$, and $337 \mathrm{mg} / \mathrm{dL})$. Thus, it is not clear whether the software recommendation was erroneous. No cases of "Different; $>20 \% "$ " (category 5 ) were noted.

Of the 103 cases categorized as "Other" (category 6), in 40 non-clustered episodes ( $1.6 \%$ of the entire data set), the study team recommended increasing a dosage, whereas the soft- ware recommended decreasing it. The rest of the "Other" cases included adjustments differing by more than $20 \%$ mostly because of quantization (e.g., if a bolus dosage component of 4 units was increased by the study team to 5 units, whereas it was kept unchanged by the software, the difference was $25 \%$ ).

In summary, $95.6 \%$ of the software recommendations (using only glucose measurements to adjust the prior dosage) yielded clinically equivalent insulin dosage adjustments when compared with those of the study team.

As illustrated by the examples in Figure 2C, in all subjects, insulin dosage adjustments were essential not only for induction of intensive insulin therapy but also for maintaining optimal A1C during the entire study.

\section{Glucose reading is the only parameter required to frequently adjust insulin dosage, invariant to insulin sensitivity}

To determine whether the similarity between recommendations of the software and the study team was not related to subjects who required either high or low insulin dosage, we divided the 2,520 data points into three groups $(820,776$, and 924, corresponding to eight, nine, and nine subjects) according to the subjects' insulin sensitivity. Insulin sensitivity was calculated by total daily insulin dose (in units) divided by weight (in $\mathrm{kg}$ ) at the end of the trial. The subjects were divided into the following groups: (A) 0.3-0.6 (21-59 units/day), (B) 0.6-1.0 (52-120 units/day), and (C) 1.0-1.9 (113-232 units/ day).

In all three groups, more than $93 \%$ of the software dosage recommendations were clinically equivalent to the study team's dosage recommendations, i.e., no more than $20 \%$ apart (Fig. 2D). Similarity between dosage recommendations tended to be lower for patients treated with fewer insulin units per day. This was ascribed to clinically minor differences of 1-2 units between the software and the study team's recommendations. In conclusion, these results suggest that frequent insulin dosage adjustments can be made based only on glucose readings independent of patients' insulin sensitivity.

\section{Other clinical guidelines for insulin dosage adjustments based on glucose readings may reasonably correlate with ones made by trained care-providers}

Our software was designed to imitate the decision making process of a care-provider when adjusting insulin dosage every 1-4 weeks. Yet, different care-providers may have different approaches. Although not initially designed to operate independent of the care-provider input, we implemented in software the guidelines for frequent insulin dosage adjustments published by Bergenstal et al. ${ }^{12}$ The same data used before from the $630 \log$ sheets $(2,520$ insulin dosage adjustments) were fed to "Bergenstal's guidelines," and the recommendations were compared to the original recommendations of the study team. In $15.8 \%$ of the cases the guidelines gave identical recommendations to the ones made by the study team; in $32.5 \%$, "within $10 \%$ "; in $39.2 \%$, "within 10-20\%"; no "different 10-20\%" or "different $>20 \%$ " were noted; and $12.5 \%$ were categorized as "other" (data not shown). 


\section{Discussion}

This report explores the vital clinical parameter required to facilitate frequent insulin dosage adjustments. Unfortunately, the growing mismatch between patients' needs and caregivers' availability disallows frequent outpatient insulin dosage adjustments, thus making insulin therapy less effective. In the preceding analysis, we compared modifications of insulin dosage (long-acting and mealtime fast-acting) made by a dedicated software to ones made by an experienced study team. The only input the software used to adjust insulin dosage was glucose readings, whereas the study team communicated with the patients and could have been exposed to other clinical parameters. We showed that the software recommendations were clinically equivalent to the ones made by the study team. Concomitantly, the original recommendations of the study team were demonstrated to be safe and effective. Although frequently adjusted insulin therapy has been supported by multiple studies, ${ }^{5-8,11-13}$ the optimal frequency of adjustments is yet to be determined. In this study, subjects were contacted every 1-4 weeks for dosage adjustments and confer superior control without excess of hypoglycemia.

The frequency of severe hypoglycemia both in the reanalyzed cohort and in the original study population was low as been routinely demonstrated elsewhere and considered to be beneficial despite its risk. ${ }^{13,14,19-21}$ In more than $80 \%$ of the cases, dosage adjustment recommendations made by the software were, at most, within $10 \%$ of those made by experienced endocrinologists and nurses. In $15 \%$ of the cases dosage modifications made by the software were within $10-20 \%$ of the study team's recommendations. Similar correlation was demonstrated separately for long-acting and fast-acting insulin and among subjects with different insulin sensitivity.

In our metric, the ability of the software to make safe insulin dosage adjustments was assessed by six distance categories (see Similarity metric). Categories 4 and 5 included cases in which the software recommended an increase in dosage whereas the study team recommended decreasing it. These categories were considered as potentially hazardous because an overdose of insulin may result in hypoglycemia. Yet, in the eight cases $(0.3 \%)$ classified as "Different; 10-20\%" (category 4) it was unclear whether following the software recommendations was unreasonable. As outlined in Figure 2A, no events of "Different; $>20 \%$ " (category 5) were identified.

Category 6, i.e., "Other," was found in $4.1 \%$ of the cases. This category included cases where the study team recommended increasing a dosage component whereas the software recommended decreasing the same component. In these cases it could be inferred that underdosing may lead to subsequent hyperglycemia, followed by overdosing and finally hypoglycemia. Our static comparison could not have unequivocally excluded it. Yet, such episodes occurred rarely (40 non-clustered cases, or $1.6 \%$ of all episodes of dosage adjustments) and were therefore unlikely to have lead to hazardous situations if the software were to independently make frequent dosage adjustments.

Although the software is not intended to operate independent of a care-provider's input, we computed clinical guidelines for insulin dosage adjustments suggested by a different institution. We found reasonable correlation implying that clinical guidelines for frequently adjusted insulin therapy based on glucose readings do not require additional parameters to make the therapy effective.

Our study is limited by the fact that subjects were closely followed and more titrated by an expert study team. Unfortunately, in reality, patients are infrequently seen in the clinic, and their insulin dosage is infrequently adjusted. Still, only clinical studies that incorporate close follow-up allow the type of analysis presented. Although the A1C goal set in our study was questioned by the ACCORD study, ${ }^{22}$ the ACCORD patients were treated with multiple diabetes medications and not only with insulin. Recent analysis of the ACCORD data did not find insulin therapy to be an independent risk factor for adverse outcome. ${ }^{23}$

The software discussed here emulates the decision-making process of an expert care-provider, coaching the patient to frequently adjust insulin dosage. Although we fully acknowledge the impact of diet and exercise on diabetes management, the presented data suggest that glucose readings alone are sufficient to enable effective and safe insulin dosage optimization provided that dosage adjustments are made frequently. A prospective clinical study where a healthcare provider uses only glucose data to adjust subjects' insulin therapy dosage is planned.

In today's reality, the overwhelming workload in clinics and the deficiency in care-providers trained in insulin titration make frequent insulin dosage adjustments unrealistic. Consequently, only $35 \%$ of insulin-treated patients achieve A1C $<7 \%$ because insulin dosage is seldom adjusted and regimens become too rigid to compensate for the dynamic needs of patients. Dependent on further clinical data, we postulate that such software has the capacity to enable patients to safely realize the full benefits of their insulin therapy by adjusting insulin dosage between appointments in order to achieve optimal glycemic control. This can alleviate the care-providers from their impossible task to frequently optimize insulin therapy, shorten clinic waiting time, and assign more time for patients' education and management of co-morbidities.

\section{Author Disclosure Statement}

Both I.H. and E.B. are co-founders of Hygieia Inc. W.H.H. declares no competing financial interests.

\section{References}

1. Hoerger TJ, Segel JE, Gregg EW, Saaddine JB: Is glycemic control improving in U.S. adults? Diabetes Care 2008;31: 81-86.

2. Koro CE, Bowlin SJ, Bourgeois N, Fedder DO: Glycemic control from 1988 to 2000 among U.S. adults diagnosed with type 2 diabetes: a preliminary report. Diabetes Care 2004;27: 17-20.

3. Schifferdecker E, Schmidt K, Boehm BO, Schatz H: Longterm compliance of intensified insulin therapy. Diabetes Res Clin Pract 1994;23:17-23.

4. Spoelstra JA, Stolk RP, Heerdink ER, Klungel OH, Erkens JA, Leufkens HG, Grobbee DE: Refill compliance in type 2 diabetes mellitus: a predictor of switching to insulin therapy? Pharmacoepidemiol Drug Saf 2003;12:121-127.

5. Holman RR, Thorne KI, Farmer AJ, Davies MJ, Keenan JF, Paul S, Levy JC; 4-T Study Group: Addition of biphasic, 
prandial, or basal insulin to oral therapy in type 2 diabetes. N Engl J Med 2007;357:1716-1730.

6. Bretzel RG, Nuber U, Landgraf W, Owens DR, Bradley C, Linn T: Once-daily basal insulin glargine versus thrice-daily prandial insulin lispro in people with type 2 diabetes on oral hypoglycaemic agents (APOLLO): an open randomised controlled trial. Lancet 2008;371:1073-1084.

7. Janka HU, Plewe G, Riddle MC, Kliebe-Frisch C, Schweitzer MA, Yki-Järvinen H: Comparison of basal insulin added to oral agents versus twice-daily premixed insulin as initial insulin therapy for type 2 diabetes. Diabetes Care 2005;28: 254-259.

8. Kennedy L, Herman WH, Strange P, Harris A: Impact of active versus usual algorithmic titration of basal insulin and point-of-care versus laboratory measurement of $\mathrm{HbA1c}$ on glycemic control in patients with type 2 diabetes: the Glycemic Optimization with Algorithms and Labs at Point of Care (GOAL A1C) trial. Diabetes Care 2006;29:1-8.

9. Buse JB, Wolffenbuttel BH, Herman WH, Shemonsky NK, Jiang HH, Fahrbach JL, Scism-Bacon JL, Martin SA: DURAbility of basal versus lispro mix 75/25 insulin efficacy (DURABLE) trial 24-week results: safety and efficacy of insulin lispro mix $75 / 25$ versus insulin glargine added to oral antihyperglycemic drugs in patients with type 2 diabetes. Diabetes Care 2009;32:1007-1013.

10. Davies M, Evans R, Storms F, Gomis R, Khunti K: Initiation of insulin glargine in suboptimally controlled patients with type 2 diabetes: sub-analysis of the AT.LANTUS trial comparing treatment outcomes in subjects from primary and secondary care in the UK. Diabetes Obes Metab 2007;9: 706-713.

11. Herman WH, Ilag LL, Johnson SL, Martin CL, Sinding J, Al Harthi A, Plunkett CD, LaPorte FB, Burke R, Brown MB, Halter JB, Raskin P: A clinical trial of continuous subcutaneous insulin infusion versus multiple daily injections in older adults with type 2 diabetes. Diabetes Care 2005;28: 1568-1573.

12. Bergenstal RM, Johnson M, Powers MA, Wynne A, Vlajnic A, Hollander P, Rendell M: Adjust to target in type 2 diabetes: comparison of a simple algorithm with carbohydrate counting for adjustment of mealtime insulin glulisine. Diabetes Care 2008;31:1305-1310.

13. Effect of intensive therapy on the microvascular complications of type 1 diabetes mellitus. JAMA 2002;287:2563-2569.

14. Holman RR, Paul SK, Bethel MA, Matthews DR, Neil HA: 10year follow-up of intensive glucose control in type 2 diabetes. N Engl J Med 2008;359:1577-1589.
15. McMahon GT, Dluhy RG: Intention to treat-initiating insulin and the 4-T study. N Engl J Med 2007;357:1759-1761.

16. Riddle MC: Starting and advancing insulin for type 2 diabetes: algorithms and individualized methods are both necessary. J Clin Endocrinol Metab 2008;93:372-374.

17. Standards of medical care in diabetes-2009. Diabetes Care 2009;32(Suppl 1):S13-S61.

18. Heinemann L: Variability of insulin absorption and insulin action. Diabetes Technol Ther 2002;4:673-682.

19. Doyle EA, Weinzimer SA, Steffen AT, Ahern JA, Vincent M, Tamborlane WV: A randomized, prospective trial comparing the efficacy of continuous subcutaneous insulin infusion with multiple daily injections using insulin glargine. Diabetes Care 2004;27:1554-1558.

20. DeVries JH, Snoek FJ, Kostense PJ, Masurel N, Heine RJ: A randomized trial of continuous subcutaneous insulin infusion and intensive injection therapy in type 1 diabetes for patients with long-standing poor glycemic control. Diabetes Care 2002;25:2074-2080.

21. Tsui E, Barnie A, Ross S, Parkes R, Zinman B: Intensive insulin therapy with insulin lispro: a randomized trial of continuous subcutaneous insulin infusion versus multiple daily insulin injection. Diabetes Care 2001;24:1722-1727.

22. Action to Control Cardiovascular Risk in Diabetes Study Group, Gerstein HC, Miller ME, Byington RP, Goff DC Jr, Bigger JT, Buse JB, Cushman WC, Genuth S, Ismail-Beigi F, Grimm RH Jr, Probstfield JL, Simons-Morton DG, Friedewald WT: Effects of intensive glucose lowering in type 2 diabetes. N Engl J Med 2008;358:2545-2559.

23. Calles-Escandón J, Lovato LC, Simons-Morton DG, Kendall DM, Pop-Busui R, Cohen RM, Bonds DE, Fonseca VA, IsmailBeigi F, Banerji MA, Failor A, Hamilton B: Effect of intensive compared with standard glycemia treatment strategies on mortality by baseline subgroup characteristics: the Action to Control Cardiovascular Risk in Diabetes (ACCORD) trial. Diabetes Care 2010;33:721-727.

Address correspondence to: Israel Hodish, M.D., Ph.D. Division of Metabolism, Endocrinology $\mathcal{E}$ Diabetes University of Michigan Brehm Tower, Room 5,321 1,000 Wall Street Ann Arbor, MI 48105

E-mail: ihodish@umich.edu 